\title{
Forum
}

\section{The welfare and conservation of Asian elephants - a reply to Sukumar}

\author{
Paul A. Rees
}

Since my summary of the global fate of Asian elephants in zoos (this issue) was written Clubb \& Mason (2002) have published a review of the welfare of zoo elephants in Europe, commissioned by the Royal Society for the Prevention of Cruelty to Animals in the UK. In an attempt to collect data on behaviour, reproduction, group composition, welfare and other aspects of husbandry, they sent questionnaires to the directors of the 18 zoos in the UK that hold elephants. Professor Sukumar doubts my contention that zoo directors lack the commitment necessary to manage the zoo elephant population as viable breeding units. Why then did none of the zoos contacted by Clubb \& Mason reply?

After an extensive review of the literature and an analysis of available population data, Clubb \& Mason concluded that the breeding and importation of elephants should be halted until the factors responsible for poor welfare have been investigated. They also recommended that only zoos that then solve these problems should be allowed to keep elephants in the future.

Clubb \& Mason's research clearly had a welfare agenda, and they did not directly address the conservation role of zoo elephants. Students of animal welfare generally take the view that it is the welfare of the individual animal that is paramount, whereas conservationists are primarily concerned with the survival of species. Threatened species, however, are not caged mink or factory-farmed poultry. Many rare species survive in the wild only because the 'rights' of their predecessors were infringed when they were taken into captive breeding programmes. If we take a purely animal welfare approach to dealing with zoo elephants we would have to treat them all as individuals and ignore the need to prevent the extinction of the species. We could look after the welfare of the individual animals to a very high standard and let the captive breeding programmes fail.

If zoos are to continue to keep elephants they need to find a compromise that both meets welfare requirements and the conservation objectives of establishing sustainable captive populations. These objectives may have synergistic solutions because addressing welfare issues may contribute to captive breeding success. The resultant presence of young animals will create more natural social structures and be much more valuable in stimulating natural behaviour than artificial enrichment devices (Rees, 2000).

Professor Sukumar proposes an ambitious global study of the potential future conservation role of captive Asian elephants. Such an analysis would be interesting, but whether or not western zoos have a part to play is debatable. These zoos are independent institutions that cooperate in breeding programmes only by consent. Some have decided to invest in new elephant facilities, but this alone does not guarantee that they will be able to obtain more elephants in the future (from the wild or from other zoos), or that these elephants will breed. Cooperative efforts are further constrained by international law (the Convention on Trade in Endangered Species of Fauna and Flora, 1973), and according to Clubb \& Mason (2002) only 67 Asian elephants were exported from Asia to European zoos between 1975 and 1999.

Those zoos which will be best placed to respond to the criticism of the animal welfare lobby and the conservation movement are likely to be those that have high husbandry standards, large breeding groups and active outreach programmes in Asia. A small number of such zoos already exist. Professor Sukumar summarizes the situation perfectly: "The maintenance of elephants in western zoos must be firmly linked to conservation efforts in the elephant's range states".

\section{References}

Clubb, R. \& Mason, G. (2002) A Review of the Welfare of Zoo Elephants in Europe. A report commissioned by the RSPCA. Animal Behaviour Research Group, Department of Zoology, University of Oxford, Oxford, UK.

Rees, P. A. (2000) Are elephant enrichment studies missing the point? International Zoo News, 47, 369-371.

Paul A. Rees School of Environment \& Life Sciences, Allerton Building

University of Salford, M6 6PU, UK. E-mail: p.a.rees@salford.ac.uk

Manuscript accepted 25 October 2002 